\title{
Langmuir
}

pubs.acs.org/Langmuir

(C) 2010 American Chemical Society

\section{Kinetic Parameters from Detection Probability in Single Molecule Force Spectroscopy}

\author{
Chad Ray, Senli Guo, Jason Brown, Nan Li, and Boris B. Akhremitchev* \\ Department of Chemistry, Duke University, Durham, North Carolina 27708
}

Received March 31, 2010. Revised Manuscript Received May 12, 2010

\begin{abstract}
The detection probability of rupture events in AFM force spectroscopy measurements presents a viable alternative to standard methods for extracting kinetic parameters of dissociation. The detection probability has a maximum as a function of the probe velocity where (1) the probability to form a molecular bond is independent of the probe velocity and (2) the detection of rupture events is limited by noise and performed with a constant density of data points per distance of the probe displacement. This newly developed model indicates that the optimal detection velocity is independent of dissociation rate and depends on the distance to the barrier kinetic parameter. Therefore, the kinetic parameters of bond dissociation can be extracted from the dependence of detection probability on probe velocity and the detection threshold. This approach is sensitive to low rupture forces and therefore is complementary to the common most probable force data analysis approach. The developed approach is tested using rupture forces measured with specific bonds between biotin and streptavidin and with nonspecific bonds between linear alkanes in water. Results for the analysis of specific bonds rupture are consistent with the previous measurements, suggesting that rupture forces spanning a wide range of values originate from the same binding potential. Kinetic parameters obtained for linear alkanes are significantly different from previous measurements suggesting possible heterogeneity of the bound state.
\end{abstract}

\section{Introduction}

Single molecule dynamic force spectroscopy quantifies the potential of mean force between molecules. Because measurements can be performed in various media and pairwise interactions can be studied directly, this experimental technique is becoming a valuable analytical tool of physicochemical and biophysical research. ${ }^{1-10}$ In these measurements, interacting molecules are connected to two opposing surfaces that are brought into contact and then separated. During separation the rupture forces at different velocities of separation are registered and analyzed by suitable theoretical models. ${ }^{2,11-19}$ The successful application of theoretical models

*To whom correspondence should be addressed. Telephone: 919-660-1648. Fax: 919-660-1605. E-mail: boris.a@duke.edu.

(1) Florin, E. L.; Moy, V. T.; Gaub, H. E. Science 1994, 264, 415-417.

(2) Evans, E.; Ritchie, K. Biophys. J. 1997, 72, 1541-1555.

(3) Izrailev, S.; Stepaniants, S.; Balsera, M.; Oono, Y.; Schulten, K. Biophys. J. 1997, 72, 1568-1581.

(4) Grandbois, M.; Beyer, M.; Rief, M.; Clausen-Schaumann, H.; Gaub, H. E. Science 1999, 283, 1727-1730.

(5) Bustamante, C.; Chemla, Y. R.; Forde, N. R.; Izhaky, D. Annu. Rev. Biochem. 2004, 73, 705-748.

(6) Ray, C.; Akhremitchev, B. B. J. Am. Chem. Soc. 2005, 127, 14739-14744.

(7) Ray, C.; Brown, J. R.; Akhremitchev, B. B. J. Phys. Chem. B 2006, 110, 17578-17583.

(8) Ray, C.; Gu, C.; Brown, J. R.; Kirkpatrick, A.; Akhremitchev, B. B. J. Phys. Chem. C 2008, 112, 18164-18172.

(9) Friddle, R. W.; Sulchek, T. A.; Albrecht, H.; De Nardo, S. J.; Noy, A. Curr. Nanosci. 2007, 3, 41-48.

(10) Liang, J.; Fernandez, J. M. ACS Nano 2009, 3, 1628-1645.

(11) Hummer, G.; Szabo, A. Biophys. J. 2003, 85, 5-15.

(12) Dudko, O. K.; Filippov, A. E.; Klafter, J.; Urbakh, M. Proc. Natl. Acad. Sci. U.S.A. 2003, 100, 11378-11381.

(13) Dudko, O. K.; Hummer, G.; Szabo, A. Phys. Rev. Lett. 2006, 96, 108101. (14) Gu, C.; Ray, C.; Guo, S.; Akhremitchev, B. B. J. Phys. Chem. C 2007, 111, 12898-12905.

(15) Hyeon, C.; Thirumalai, D. J. Phys. Condens. Matter 2007, 19, 113101.

(16) Gu, C.; Kirkpatrick, A.; Ray, C.; Guo, S.; Akhremitchev, B. B. J. Phys.

Chem. C 2008, 112, 5085-5092.

(17) Friddle, R. W. Phys. Rev. Lett. 2008, 100, 138302

(18) Walcott, S. J. Chem. Phys. 2008, 128, 215101

(19) Ho, D.; Zimmermann, J. L.; Dehmelt, F. A.; Steinbach, U.; Erdmann, M.; Severin, P.; Falter, K.; Gaub, H. E. Biophys. J. 2009, 97, 3158-3167. requires that the rupture events detected in experiments mainly come from the ruptures of individual physical bonds. The detection of interactions between individual molecules requires sparse attachment of the molecules to the interacting surfaces. Therefore, over many contacts between the surfaces, the detection of a bond rupture should be an infrequent event. The probability to form a molecular bond depends on grafting density at both surfaces, on rate of association reaction, and on contact time available to establish a molecular bond. ${ }^{20}$ Analytical models have been developed to predict binding probability for tethered molecules that are free to diffuse in solution..$^{20,21}$ On the basis of these models, it might be expected that the detection probability of rupture events should be independent of the probe velocity if the interacting molecules are adsorbed to the surfaces and cannot explore the solution volume near the attachment point. ${ }^{20}$ In contrast to this expectation, our force spectroscopy experiments that use hydrophobic alkanes tethered by water-soluble linkers indicate that the probability of detection of individual ruptures as a function of the probe velocity is bell-shaped. ${ }^{22}$ In this article, a model is proposed for the dependence of the detection probability on the probe velocity for the surface-adsorbed molecules and for experiments when the probe spends a relatively long time in contact with the surface. Predictions by this model are consistent with the observed dependencies. The developed analytical model relates kinetic parameters of the dissociation reaction and the detection probability dependence on probe velocity and on force detection threshold. This method of analysis is sensitive to low rupture forces and therefore it complements the common data analysis method that uses the most probable rupture forces. ${ }^{2}$ Consequently we can probe whether measured rupture forces

(20) Guo, S.; Lad, N.; Ray, C.; Akhremitchev, B. B. Biophys. J. 2009, 96, 3412-3422.

(21) Hinterdorfer, P.; Baumgartner, W.; Gruber, H. J.; Schilcher, K.; Schindler, H. Proc. Natl. Acad. Sci. U.S.A. 1996, 93, 3477-3481.

(22) Ray, C.; Brown, J. R.; Kirkpatrick, A.; Akhremitchev, B. B. J. Am. Chem. Soc. 2008, 130, 10008-10018. 


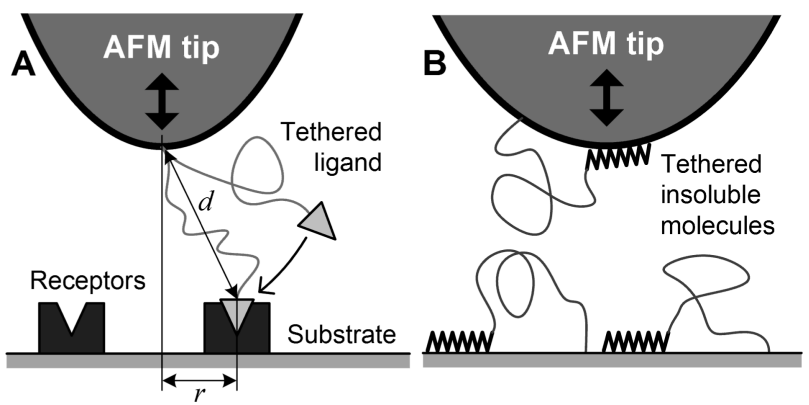

Figure 1. (A) Binding of a soluble ligand molecule tethered at the AFM probe to a receptor immobilized on the substrate; (B) binding between insoluble tethered molecules.

originate from the same potential of mean force or from different potentials thus reporting on heterogeneity of the system under study. The developed model is used to fit the detection probabilities and to extract kinetic parameters of ruptures between specific biotin-streptavidin receptor-ligand bond and nonspecific interactions between linear alkanes in water. Comparison with the previously reported parameters indicates that this detection probability-based data analysis approach in combination with other currently used methods might reveal binding heterogeneity even when distributions of rupture forces significantly overlap.

\section{Theoretical Model}

In this section we develop a model of the detection probability of rupture events. We then show how the noise-limited detection probability can be used to extract kinetic parameters of dissociation.

2.1. Binding Probability of Tethered Molecules. Consider a case where a tethered molecule (a ligand) is free to move in solution and during its diffusive motion might encounter a receptor molecule bound to the substrate as illustrated in Figure 1A. It has been suggested that for such a case the binding probability during one approach-withdraw cycle of the AFM probe can be estimated by ${ }^{20}$

$$
p(r)=1-\exp \left[-\left(k(r) t_{\mathrm{dwell}}+2 \int_{0}^{z_{0} / v} \mathrm{~d} \tau k(d(\tau))\right)\right]
$$

Here $r$ is the displacement of the receptor from the projection of the fixed tether end onto the substrate (see Figure 1A), $k(d)$ is the distance-dependent rate of the receptor-ligand bonding, $t_{\text {dwell }}$ is the dwell time the probe spends in contact with the surface, $d$ is the time-dependent distance between the receptor and the attachment point of a ligand, $\tau$ is time of up-down probe motion, $z_{0}$ is the maximum probe distance from the substrate where the receptorligand binding is still thermodynamically favorable, and $v$ is the probe velocity. Here we assume that the bond lifetime significantly exceeds $\left(t_{\text {dwell }}+2 z_{0} / v\right)$; the factor of 2 on the right-hand side of eq 1 indicates that binding might occur during the approach or withdraw motion of the probe.

For short tethers, the binding probability depends on the rate of association and is independent of the probe velocity. ${ }^{21}$ Therefore, for experiments with insoluble molecules (see Figure 1B for illustration) binding probability is expected to be the same for different probe velocities. In addition, the time that the tip rests on the surface has two components: $t_{\text {dwell }}=t_{0}+2 \delta / v$ where $t_{0}$ is the time that the probe is intentionally resting on the surface and the second component comes from the time required to reach the predefined deflection trigger $\delta$. It has been shown that if $t_{0}$ is sufficiently long then the binding probability becomes independent of the probe velocity. ${ }^{20}$ Below we consider these cases and therefore assume that binding probability is independent of probe velocity. Binding probability is not measured in experiments directly. It is modulated by the probability of detecting a rupture event. This probability is considered in the section below.

2.2. Detection Limit. Here we assume that a molecular bond is formed between molecules attached to the surface and to the AFM probe. Whether the resulting rupture force is detected depends on the noise level. We consider the detection threshold for measuring rupture forces $\Phi=\xi \cdot \Delta F$. Here $\Delta F$ is the rms force noise and $\zeta$ is a signal-to-noise (SNR) ratio that is necessary for the accurate fitting of the stretching part of the data to the polymer model. ${ }^{23}$ The rms force noise contains the instrument white and DC noise and cantilever thermal noise components. Therefore the force threshold can be written as ${ }^{24,25}$

$$
\Phi=\varsigma\left(B\left[\frac{4 k_{\mathrm{B}} T \kappa_{\mathrm{c}}}{\omega_{0} Q}+\Delta F_{\text {white }}^{2}\right]+\Delta F_{\mathrm{DC}}^{2}\right)^{1 / 2}
$$

Here $k_{\mathrm{B}} T$ is the thermal energy, $\kappa_{\mathrm{c}}$ is the cantilever spring constant, $B$ is the detection bandwidth, $\omega_{0}$ is the angular resonant frequency of the cantilever, $Q$ is the quality factor, $\Delta F_{\text {white }}$ is the white noise, and $\Delta F_{\mathrm{DC}}$ is the DC noise. In an ideal instrument white noise and DC noise are equal to zero, however in actual measurements they differ from zero and can be determined experimentally.

The data are often recorded by approximately maintaining the density of data points per displacement distance of the probe. Therefore, the detection bandwidth becomes a function of the probe velocity $v$ and the density of data points $D_{\mathrm{N}}$ recorded per unit distance. Thus eq 2 becomes ${ }^{23}$

$$
\Phi=\varsigma \cdot\left[\frac{v \cdot D_{\mathrm{N}}}{2}\left(\frac{4 k_{\mathrm{B}} T \kappa_{\mathrm{c}}}{\omega_{0} Q}+\Delta F_{\text {white }}{ }^{2}\right)+\Delta F_{\mathrm{DC}}{ }^{2}\right]^{1 / 2}
$$

The rupture force of an individual bond is a stochastic property. Initially we examine the most probable rupture forces for the simplicity of the argument. The most probable rupture force $F^{*}$ of an individual bond is approximately proportional to the logarithm of the probe velocity. ${ }^{2,26}$ Microscopic models ${ }^{11,12}$ predict a deviation of $F^{*}$ from the logarithmic dependence, however this deviation is usually small in AFM-based measurements. ${ }^{14}$ Comparison of the $\log (v)$ function with the dependence given by eq 3 immediately suggests that if the $F^{*}$ is above the noise threshold at some probe velocity, it will fall below that threshold for sufficiently low and high velocities. Figure 2 compares the noise threshold computed by eq 3 , using realistic parameters, shown in the figure, and the most probable forces, computed by a model that takes into account polymeric tether stiffening by using the freely jointed chain (FJC) model ${ }^{27}$ Comparison of the detection threshold $\Phi$ and the most probable rupture forces $F^{*}$ computed for different dissociation rates confirms the above suggestion about the noise thresholds in rupture force detection. Also shown in the figure is a noise threshold for the rupture force

(23) Guo, S.; Ray, C.; Kirkpatrick, A.; Lad, N.; Akhremitchev, B. B. Biophys. J 2008, 95, 3964-3976.

(24) Viani, M. B.; Schaffer, T. E.; Chand, A.; Rief, M.; Gaub, H. E.; Hansma, P. K. J. Appl. Phys. 1999, 86, 2258-2262.

(25) Sarid, D. Scanning Force Microscopy; Oxford University Press: New York, 1994.

(26) Evans, E.; Ritchie, K. Biophys. J. 1999, 76, 2439-2447.

(27) Ray, C.; Brown, J. R.; Akhremitchev, B. B. J. Phys. Chem. B 2007, 111, 1963-1974. 


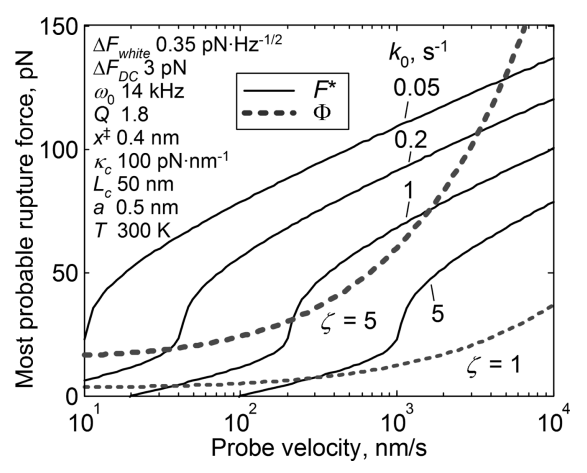

Figure 2. The noise threshold and most probable forces computed for four values of the dissociation rate $k_{0}$. Calculation parameters are shown in the figure. Note the log scale on the abscissa axis.

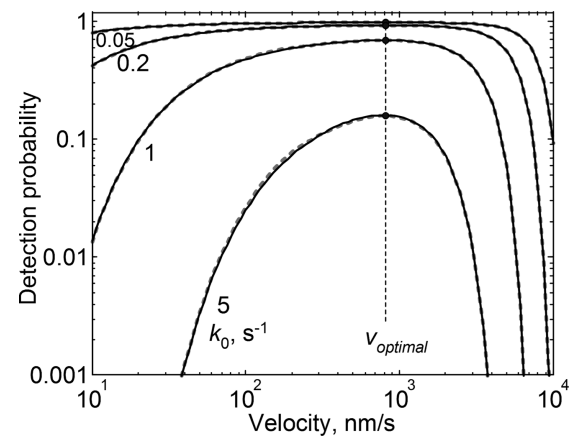

Figure 3. Relative detection probability of rupture events for bonds with different dissociation rates. Dissociation rates are indicated below the corresponding curves. Calculations using the FJC tether model are shown with solid lines and calculations using the approximate FJC model (Supporting Information eq S1) are shown with dashed lines (see text for more details).

detection with the $\operatorname{SNR} \xi=1$. It can be noted that the most probable forces are mostly above this threshold. However measurement of the loading rate by linear fitting at the rupture point requires that a relatively short part of a force curve is analyzed because of the nonlinear tether elasticity. Therefore low SNR in the force detection will result in large error in measuring the loading rate. This error can be decreased by increasing the number of acquired data points, but such improvement is limited by the noise that increases when the detection bandwidth approaches the resonance frequency of the cantilever. ${ }^{25}$ Therefore we use a SNR higher than 5 to accurately measure the loading rate.

To estimate the noise effects on the detection probabilities, we need to consider the probability density of rupture forces. The rupture forces below the noise threshold will remain undetected. The probability to detect rupture forces is therefore proportional to

$$
p_{\mathrm{D}}=1-\int_{0}^{\Phi} p(F) \mathrm{d} F=S(\Phi)
$$

Here $p_{D}$ is the probability to detect the bond rupture at the noise threshold $\Phi, p(F)$ is the probability density function of rupture force $F$, and $S$ is the bond survival probability. Figure 3 shows the detection probabilities calculated using eq 4 using the same model and parameters as in Figure 2.

2.3. Optimal Detection Velocity. Calculations shown in Figure 3 indicate that the detection probability has a maximum value as a function of a probe velocity and it decreases rapidly for high probe velocities. The significant decrease in the detection probability limits the range of useful high velocities below the limit determined by the drag forces. ${ }^{28}$

By setting $\mathrm{d} p_{\mathrm{D}} / \mathrm{d} v=0$ the velocity of the maximum detection probability can be determined (henceforth called the optimal detection velocity). We consider dissociation reactions described by the first-order kinetics where dependence of the survival probability on the pulling force is ${ }^{29}$

$$
S(F)=\exp \left(-\int_{0}^{F} \mathrm{~d} f k(f) / v_{\mathrm{F}}(f)\right)
$$

Here $k(F)$ is the force-dependent dissociation rate of a molecular bond, and $v_{\mathrm{F}}(F)=\mathrm{d} F / \mathrm{d} t$ is the loading rate. For pulling experiments with polymeric tethers the loading rate is $v_{\mathrm{F}}(F)=v /\left(\kappa_{\mathrm{c}}{ }^{-1}+\right.$ $\left.l^{\prime}(F)\right)$ where $v$ is the pulling velocity, $\kappa_{\mathrm{c}}$ is the spring constant of a force sensor, and $l(F)$ is the force-dependent length of a tether; the prime denotes differentiation with respect to force. ${ }^{14}$ Therefore, the optimal detection velocity $v_{0}$ can be obtained by solving the transcendental equation:

$$
v_{0}=\frac{\int_{0}^{\Phi\left(v_{0}\right)} k(F)\left[\kappa_{\mathrm{c}}^{-1}+l^{\prime}(F)\right] \mathrm{d} F}{k\left(\Phi\left(v_{0}\right)\right)\left[\kappa_{\mathrm{c}}^{-1}+l^{\prime}\left(\Phi\left(v_{0}\right)\right)\right]}\left(\frac{\mathrm{d} \Phi}{\mathrm{d} v}\right)_{v=v_{0}}^{-1}
$$

Here the dependence of the noise threshold $\Phi$ on probe velocity is given by eq 3 . Using a general expression for the force-dependent dissociation $\operatorname{rate}^{30} k(F)=k_{0} \int_{0}^{F} \mathrm{~d} f x^{\star}(f) /\left(k_{\mathrm{B}} T\right)$, it can be noted that the optimal detection velocity is independent of the dissociation rate at zero force $k_{0}$. In this section only the Bell-Evans model for the dissociation rate dependence on force is considered for simplicity. ${ }^{2}$ According to this model, $k(F)=$ $k_{0} \exp \left(F / F^{*}\right)$, where $F^{*}=k_{\mathrm{B}} T / x^{*}$ and $x^{*}$ is a force-independent distance between the equilibrium and the transition states. We note that other dependencies of the dissociation rate on force can be considered. ${ }^{11-13,17,30}$ If the tether is nearly linear (that is, if $\kappa_{\mathrm{c}}^{-1}+l^{\prime}(F) \approx$ const) then using eqs 3 and 6 we obtain a simpler transcendental equation that can be solved recursively:

$$
v_{0}=\frac{4 F^{\ddagger} \Phi\left(v_{0}\right)\left(1-\mathrm{e}^{-\Phi\left(v_{0}\right) / F^{\ddagger}}\right)}{\varsigma^{2} D_{\mathrm{N}}\left(\frac{4 k_{\mathrm{B}} T \kappa_{\mathrm{c}}}{\omega_{0} Q}+\Delta F_{\text {white }}{ }^{2}\right)}
$$

In addition, if the $\Delta F_{\mathrm{DC}}$ noise component in eq 3 is negligible, then the solution of eq 7 is equivalent to the solution of the transcendental equation $\Phi\left(v_{0}\right) / F^{\star}=2\left(1-\exp \left(-\Phi\left(v_{0}\right) / F^{\star}\right)\right)$ that has an approximate solution of $F^{*} \approx 0.62750 \Phi\left(v_{0}\right)$. Therefore, in the linear tether approximation and with negligible DC noise (white noise still can be included in $\Phi$ ) and with the empirical BellEvans dissociation rate dependence, the barrier width $x^{*}$ can be directly determined from the optimal detection velocity $v_{0}$ as

$$
x^{\star}=1.5936 k_{\mathrm{B}} T \Phi\left(v_{0}\right)
$$

Equation 8 provides a convenient method of estimating the barrier width; however, application of this equation to measurements performed with nonlinear polymeric tethers might produce noticeable systematic error. ${ }^{27}$ For example, optimal velocity of $810 \mathrm{~nm} / \mathrm{s}$ can be determined from plots shown in Figure 3. Using this value in eq 8 gives the barrier width of 0.61 , a $50 \%$ overestimate of the original barrier width. Neglect of the tether effects

(28) Kuhner, F.; Gaub, H. E. Polymer 2006, 47, 2555-2563.

(29) Garg, A. Phys. Rev. B 1995, 51, 15592-15595.

(30) Dudko, O. K.; Hummer, G.; Szabo, A. Proc. Natl. Acad. Sci. U.S.A. 2008, $105,15755-15760$. 


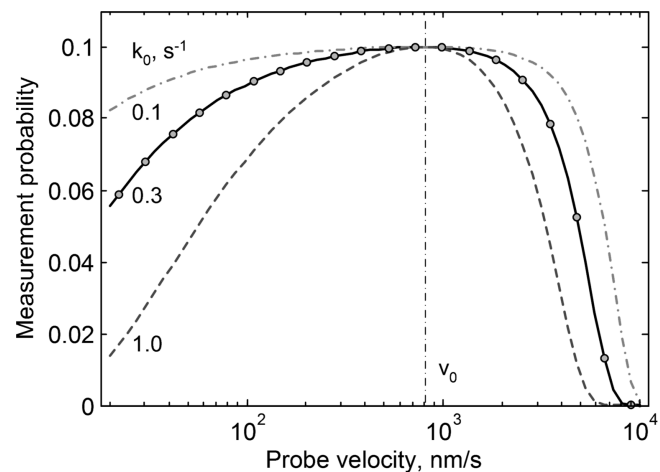

Figure 4. Probability to measure rupture events calculated for three different values of dissociation rate (shown in the graph) and scaled to the same maximum value. Vertical dash-dotted line indicates the optimal detection velocity. Calculation parameters are the same as in Figure 2.

and of the DC noise both contribute to this large inaccuracy. A significantly more accurate result can be obtained when a nonlinear model of tether elasticity is employed and all noise sources are considered. We note that analytical integration in eq 6 cannot be performed when the freely jointed chain (FJC) model of tether elasticity is used for $l(F)$. An approximation to the FJC model that allows integration in eq 6 can be used to obtain the recursive equations for the optimal detection velocity and for extraction of the barrier width. The resulting equations are cumbersome and therefore included in Supporting Information. Calculations of the detection probability using the exact FJC model and approximate FJC model (Eq. S1 in the Supporting Information file) are compared in Figure 3. Calculations by these models show little variation.

\subsection{Extracting Kinetic Parameters from Detection} Probability. As noted above, the optimal detection velocity approach permits extraction of the barrier width but does not provide information about the dissociation rate. However, the dissociation rate affects the shape of the detection probability dependence on velocity and this dependence can be used to extract both $x^{\star}$ and $k_{0}$ parameters. Probability to measure a rupture event depends on the product of detection probability and binding probability. If the binding probability is the same for all probe velocities (as discussed in section 2.1), then the probability to measure rupture events $p_{\mathrm{M}}$ can be used in the least-squares regression analysis that minimizes error $E$ :

$$
E=\sqrt{\sum_{i}\left(p_{\mathrm{M}_{i}}-\varepsilon p_{\mathrm{D}}\left(v_{i}\right)\right)^{2}}
$$

Here, the sum is taken over all probe velocities and function $p_{\mathrm{D}}$ is a function of kinetic parameters as provided by eqs 4 and 5 ; $\varepsilon$ is the binding probability. Figure 4 shows that the dependence of $p_{M}$ on probe velocity for different dissociation rates does not overlap at probe velocities away from the optimal detection velocity $v_{0}$. Therefore both $x^{*}$ and $k_{0}$ can be extracted from nonlinear leastsquares regression analysis. Fitting of the binding probability $\varepsilon$ can be avoided by normalizing probabilities by the mean value:

$$
E=\sqrt{\sum_{i}\left(p_{\mathrm{M}_{i}} /\left\langle p_{\mathrm{M}}\right\rangle-p_{\mathrm{D}}\left(v_{i}\right) /\left\langle p_{\mathrm{D}}\right\rangle\right)^{2}}
$$

Moreover, using this regression analysis does not require that all the data are collected with the same density of points $D_{\mathrm{N}}$. Therefore data measured with somewhat different $D_{\mathrm{N}}$ values can be used in analysis, simplifying the methodology of experiments. In addition, the sum in eqs 9 and 10 can include results obtained by using different values of signal-to-noise ratio. We use this approach in analysis of experimental data as described below.

2.5. Multiple Bond Ruptures. Several bond ruptures can be measured during one pulling experiment. ${ }^{31}$ If these ruptures are independent events occurring in parallel, all of these events should be included in the measured probability $p_{\mathrm{M}}$. (Consequently $p_{\mathrm{M}}$ and $\varepsilon$ can be larger than unity.) In double-tether experiments ${ }^{6,32}$ only events at the sip-sample separation corresponding to stretching of two tethers should be included in $p_{\mathrm{M}}$. Also in experiments that use only one tether (for example, to attach molecules to the probe) only ruptures occurring after the probe disengages from the substrate should be considered. However, multiple bonds can be ruptured within a single rupture event. ${ }^{16,23}$ Multiple bonds rupture at forces higher than a single bond. Therefore the detection probability with such multiple bonds present in the measurement will be different than the detection probability with only single bonds. Consequently, multiple ruptures might offset the extracted kinetic parameters. To account for multiple ruptures, the survival probability in eq 4 can be calculated to explicitly include the two-bond rupture events using the previously developed model as briefly described below. ${ }^{16,23}$

\section{Materials and Methods}

3.1. Sample Preparation. Probability to detect rupture events was analyzed for data collected with two different types of samples. In one set of experiments we tested specific receptor-ligand interactions between biotin and streptavidin. Here streptavidin molecules were covalently immobilized on the glass coverslip substrate and biotin molecules were covalently attached to the silicon nitride probe with poly(ethylene glycol) linkers with molecular mass of 3400 Da (PEG3400). In another set of measurements, hydrophobic interactions between alkanes in water have been studied. In these measurements, linear alkanes with 14, 16 , and 18 carbon atoms were tethered at one end of the alkane chain to the substrate and to the AFM probe (double-tether approach). ${ }^{7,22}$ Tethering was performed using water-soluble PEG3400 linkers. Sample preparation procedures and experimental procedures are described in detail elsewhere. ${ }^{7,22,23}$

3.2. Data Analysis. Rupture probabilities were obtained by automated analysis. In this analysis, data points in the measured deflection versus displacement dependence were averaged to produce approximately the same data point density $\left(\sim 1 \mathrm{~nm}^{-1}\right)$. Then the data were converted to force versus separation data where compressive force is positive and tensile force is negative. After this the point-to-point difference between force values was calculated, and adjacent regions with positive values of the pointto-point difference were joined together. This produced a large number of possible candidates for the rupture transitions. These candidates were filtered using a force threshold value that was determined as a multiple of the force noise $\Delta F$. Next the distance filter was applied to the remaining events: only the rupture events that occurred in a predetermined range of force-sample separation values were counted (see Figure 5). For biotin-streptavidin data collected using the single-tether arrangement, we used a range from 10 to $50 \mathrm{~nm}$ as a distance filter. For data collected with alkane samples in a double-tether arrangement, we used a range from 35 to $70 \mathrm{~nm}$ as a distance filter. For force thresholds, we used different values corresponding to the SNR from 7 to 16 . These SNR values usually correspond to force thresholds between $\sim 15$ and $130 \mathrm{pN}$. Because of the high threshold values no events

(31) Bemis, J. E.; Akhremitchev, B. B.; Walker, G. C. Langmuir 1999, 15, 2799-2805.

(32) Ratto, T. V.; Langry, K. C.; Rudd, R. E.; Balhorn, R. L.; Allen, M. J.; McElfresh, M. W. Biophys. J. 2004, 86, 2430-2437. 


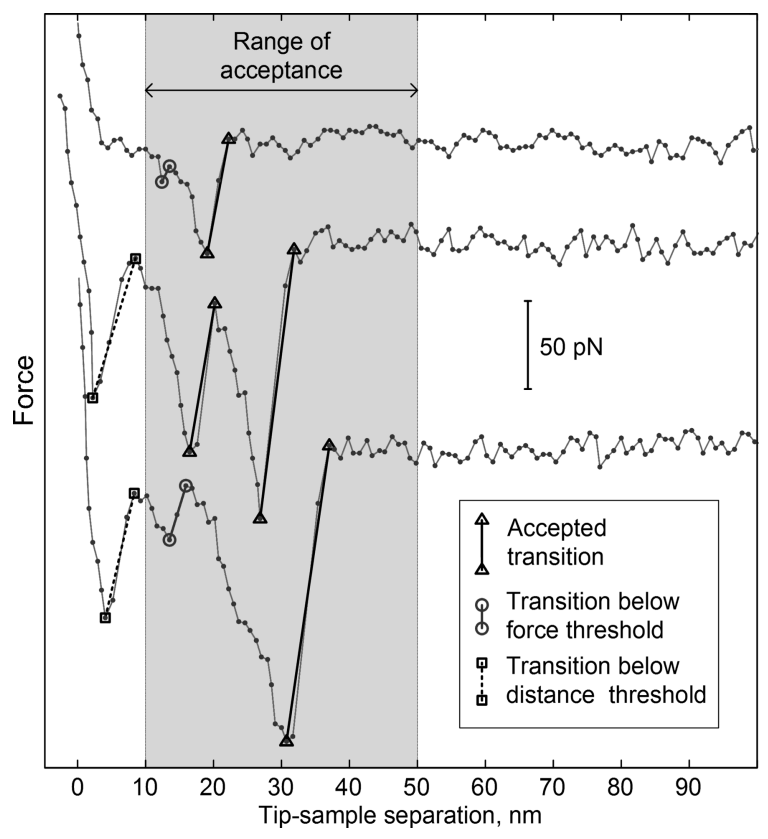

Figure 5. Force plots with automatically selected rupture events. Both accepted and rejected transitions are shown as indicated by the legend. Here the SNR for the rupture events filter equals 11. Force plots are offset for clarity. The data shown here were collected with a biotin-streptavidin sample at a probe velocity of $770 \mathrm{~nm} / \mathrm{s}$ using a cantilever with a spring constant of $56 \mathrm{pN} / \mathrm{nm}$.

resulting from random noise were expected to contribute to the counted events. This was confirmed by applying the force threshold filter to parts of force curves that are sufficiently far from the surface. A more elaborate procedure for automated selection of rupture events is necessary to include rupture events with considerably lower values of SNR. The measured rupture probability was determined by dividing the number of rupture events remaining after the filtering by the total number of force curves. The normalized rupture probability is then calculated by dividing the measured rupture probability by average rupture probability collected with different probe velocities and analyzed with different threshold levels.

The differential force values that were compared to the threshold values were produced by joining the adjacent regions with the same slope, as indicated above. Consequently, the random noise contributes to the differential force values. Therefore the threshold values $\Phi$ to be used with eqs 4 and 5 were obtained from the threshold SNR values $\xi_{\text {th }}$ (in units of the rms noise) by $\Phi=\left(\xi_{\text {th }}-\right.$ $\left.2^{1 / 2}\right) \Delta F$, where $\Delta F$ is the measured rms noise. This procedure eliminates the noise contribution to the rupture force data and helps to avoid the associated artifacts. ${ }^{33}$

Calculation of the survival probability using eq 5 requires selection of a physical model to calculate tether stretching. Because we used PEG tethers the extended FJC model was employed. ${ }^{7,34}$ This model considers the force-driven transition between helical and planar conformation of PEG chain. For data collected using the single- and double-tether approaches we used a different number of PEG units corresponding to the actual PEG used here: 80 and 160 monomers, respectively. All other parameters were the same as in the original publication. ${ }^{34}$

Measured dependencies of $p_{\mathrm{M}}$ on probe velocity and force threshold values were fit by minimizing the least-squares error given by eq 10 . The detection probability $p_{\mathrm{D}}$ on the right-hand side of eq 10 was calculated as a sum of detection probabilities for

(33) Li, N.; Guo, S.; Akhremitchev, B. B. ChemPhysChem 2010, DOI: 10.1002/ cphc.201000251, in press.

(34) Oesterhelt, F.; Rief, M.; Gaub, H. E. New J. Phys. 1999, 1, 6.1. one- and two-bond ruptures. Then according to eq $4 p_{\mathrm{D}}$ is written as

$$
p_{\mathrm{D}}(v, F)=f_{1} S(v, F)+\left(1-f_{1}\right) S\left(v, F_{1}\right) S\left(v, F-F_{1}\right)
$$

where the first term on the right-hand side corresponds to ruptures of one bond and the second term corresponds to ruptures of two bonds; $f_{1}$ is the relative probability of one-bond rupture; $F_{1}$ is the force on a shorter tether in two-bond arrangement. This force is calculated using the high-force asymptote of the FJC model as described previously. ${ }^{16}$ For calculating one-bond survival probability according to eq 5 three kinetic models were considered: the Bell-Evans model (below called "B-E" model), ${ }^{2}$ model of the paraboloidal potential with the cusp barrier ${ }^{11,13}$ (below called the "cusp" model) and model of the cubic-linear potential ${ }^{12,13}$ (below called the "cubic" model). These models provide different dependencies of the bond dissociation rates on pulling forces by making different assumptions about the shape of the potential of mean force. Calculation of the survival probability that includes effects of polymeric tethers and effects of different kinetic models is described previously ${ }^{16,23}$ and used here without change. In previous applications of the two-bond model we have considered overall force exerted by a force sensor at the moment of rupture. Here we use values of a "jump force": the difference between the force values at the beginning and at the end of rupture transition. ${ }^{35}$ Consequently in application of the two-bond model to fit experimental data the maximum relative difference between lengths of two tethers ${ }^{16}$ was kept constant at a relatively small value of 0.05 .

\section{Results and Discussion}

Typical force curves with automatically selected rupture events are shown in Figure 5. Examples of transitions that are accepted or rejected during data analysis are shown by different symbols as indicated in the legend. The majority of analyzed force curves $(>90 \%)$ did not contain events that pass through the threshold filters described in section 3.2, while some force plots contained more than one event as indicated in the graph.

Normalized rupture probabilities for two separate biotinstreptavidin experiments were fit by eq 10. The least-squares fitting was performed using three kinetic models. Examples of data and fit curves are shown in Figure 6. Extracted kinetic parameters are included in Table 1. For comparison this table also includes kinetic parameters obtained previously using the histogram fitting method. ${ }^{23}$ It has been proposed that all kinetic parameters can be obtained from force spectroscopy measurements independently. ${ }^{13}$ However, we find that for data collected with a limited range of loading rates only $x^{\star}$ and $k_{0}$ (or the barrier height $\Delta G^{\star}$ ) can be fitted independently. The barrier height $\Delta G^{*}$ and the dissociation rate are connected by equation $k_{0}=A \exp \left(-\Delta G^{*} /\left(k_{\mathrm{B}} T\right)\right)$, where $A$ is the Arrhenius pre-exponential factor. In our calculations for biotin-streptavidin interactions we used $A=10^{11} \mathrm{~s}^{-1}$, as previously. ${ }^{23,36}$ However, values in the range $10^{9}-10^{10} \mathrm{~s}^{-1}$ for the pre-exponential factor has been reported. ${ }^{37}$ Therefore, random errors for the barrier height $\Delta G^{*}$ given in Table 1 are probably lower that the systematic error arising from uncertainty in $A$. The barrier width parameters $x^{*}$ obtained here for different samples and values reported previously are well within errors of the measurements. Therefore we think that kinetic parameters extracted from analysis of detection probability are related to the

(35) Averett, L. E.; Schoenfisch, M. H.; Akhremitchev, B. B.; Gorkun, O. V. Biophys. J. 2009, 97, 2820-2828.

(36) Pincet, F.; Husson, J. Biophys. J. 2005, 89, 4374-4381.

(37) Merkel, R.; Nassoy, P.; Leung, A.; Ritchie, K.; Evans, E. Nature 1999, 397, $50-53$. 

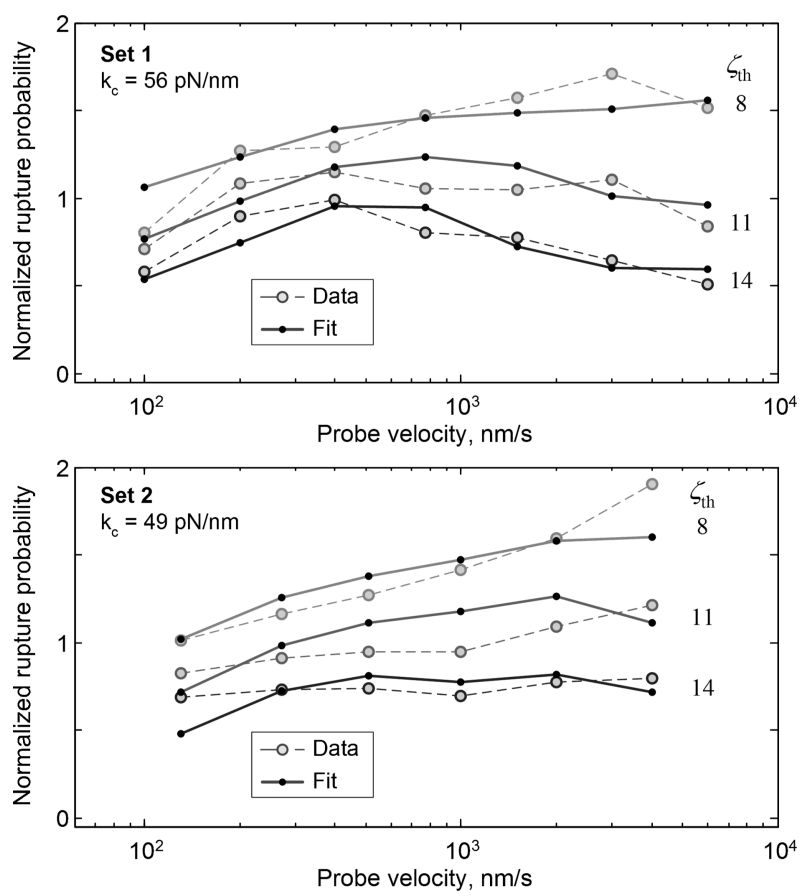

Figure 6. The normalized biotin-streptavidin rupture probabilities for two data sets and fits by eq 10 using the model of paraboloidal potential with a cusp barrier. Only three detection threshold values are shown for clarity. Thresholds of the force filter in units of SNR are shown to the right of the graphs. Parameters extracted using different kinetic models are reported in Table 1.

Table 1. Comparison of Kinetic Parameters for Biotin-Streptavidin Dissociation from This Work and Results Obtained by Histogram Fitting ${ }^{a}$

\begin{tabular}{lcccc}
\hline \multicolumn{1}{c}{ ref } & \multicolumn{2}{c}{$\mathrm{x}^{\ddagger}[\mathrm{nm}]$} & $\Delta G^{\ddagger}\left(\mathrm{kJ} \cdot \mathrm{mol}^{-1}\right)^{b}$ \\
\hline this work (B-E) $^{c}$ & $0.46 \pm 0.14$ & $0.42 \pm 0.13$ & $60.9 \pm 1.9$ & $61 \pm 2$ \\
this work (cubic) $^{c}$ & $0.49 \pm 0.14$ & $0.45 \pm 0.12$ & $61.0 \pm 1.5$ & $61 \pm 2$ \\
this work (cusp) $^{c}$ & $0.50 \pm 0.14$ & $0.46 \pm 0.12$ & $61.1 \pm 1.4$ & $61 \pm 2$ \\
histogram fit (B-E) $^{21}$ & $0.40 \pm 0.05$ & & $66.4 \pm 1.6$ & \\
histogram fit (cusp) $^{21}$ & $0.44 \pm 0.06$ & & $66.0 \pm 1.6$ &
\end{tabular}

${ }^{a}$ Reported errors calculated using the covariance matrix. ${ }^{b}$ The Arrhenius pre-exponential factor is assumed to be $10^{11} \mathrm{~s}^{-1}$. ${ }^{c}$ Results of two separate measurements are shown.

same bound state of the molecules as probed in the histogram analysis of all rupture forces. ${ }^{23}$

The same data analysis (though with different distance filter as described in section 3.2) was performed with the velocity dependence of rupture probability measured for three alkane samples. Examples of normalized rupture probability data and fit curves are shown in Figure 7. Extracted kinetic parameters are included in Table 2. There we also show kinetic parameters obtained previously using the histogram fitting method. ${ }^{22}$

Results in Table 2 show that kinetic parameters extracted for hydrophobic interactions between alkanes using the detection probability are considerably different from kinetic parameters extracted using the histogram fitting method. For the tetradecane sample the barrier width $x^{*}$ is considerably lower than that reported previously while for hexadecane and octadecane samples $x^{*}$ is considerably higher. This contrasts the observations made above about the similarity in the two analysis methods for specific biotin-streptavidin interactions.

The detection probability method developed in this work is sensitive to the decrease in probability of occurrence of low rupture force events. If measured rupture forces report on unbinding
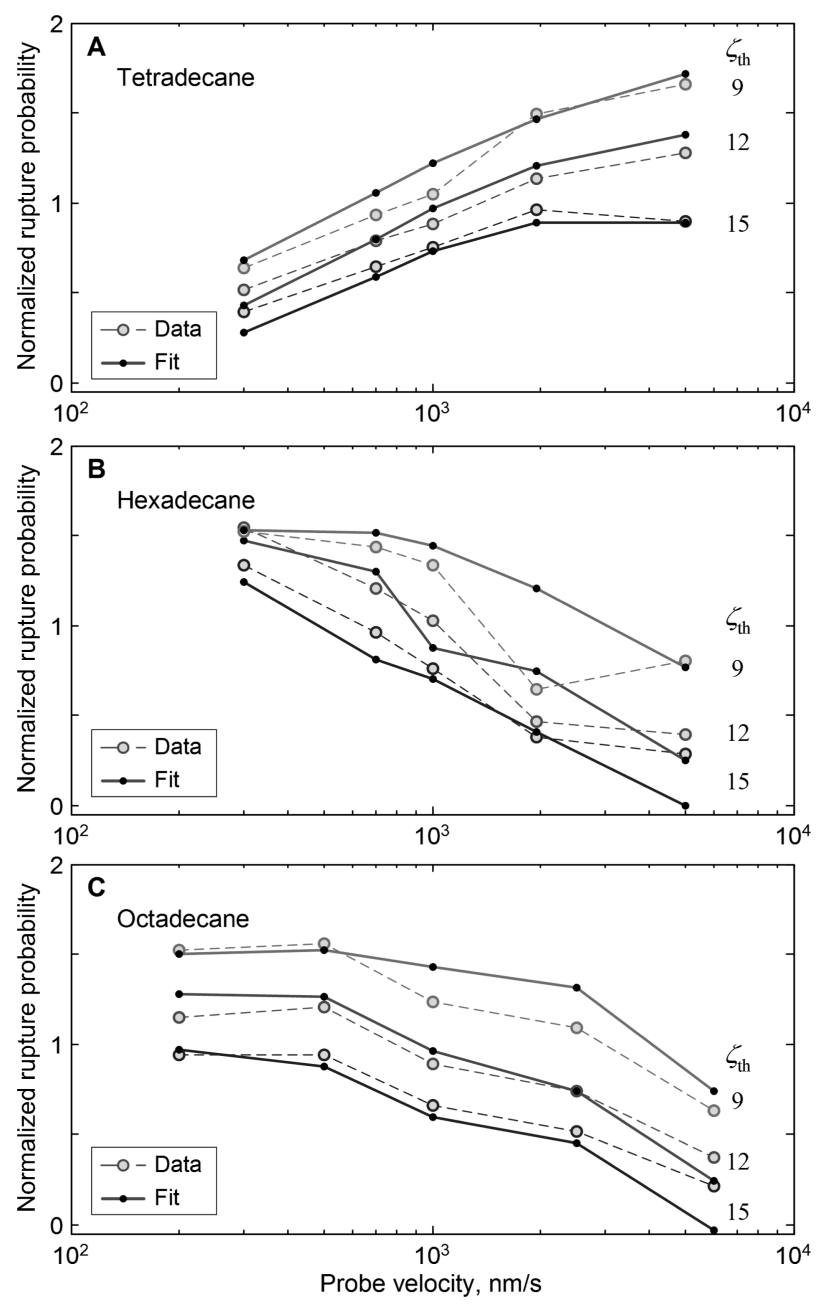

Figure 7. The normalized rupture probabilities for three alkane samples and fits by eq 10 using the model of paraboloidal potential with a cusp barrier. Only three detection threshold values are shown for clarity. Thresholds of the force filter in units of SNR are shown to the right of the graphs. Parameters extracted using different kinetic models are reported in Table 2.

from a unique free energy well, then kinetic parameters extracted using this method should be similar to kinetic parameters extracted by the histogram fitting method. However, if kinetic parameters obtained by two methods are different, then this might be an indication that the bound state prior to the rupture is heterogeneous. In the data analysis approach by Raible et al, large heterogeneity in the barrier width $x^{t}$ is used to explain the appearance of the high force tail in the distribution of rupture forces. ${ }^{38}$ We note that the two-bond model that we applied in our data analysis satisfactory explains the appearance of the high rupture forces in the distributions as discussed earlier. ${ }^{16,22,23}$ Therefore, the heterogeneity of bonds between alkanes inferred here likely originates from heterogeneity in the binding state of the individual single bond probed and not from heterogeneity in a number of bonds broken during a single rupture event. Such heterogeneity of the bound state likely originates from the occurrences of rupture events from various structurally distinct arrangements of alkane molecules in the dimer. Previously, we have suggested that linear alkanes with the chain length in the range used in this work collapse upon dimerization. ${ }^{8,22}$ Results

(38) Raible, M.; Evstigneev, M.; Bartels, F. W.; Eckel, R.; Nguyen-Duong, M.; Merkel, R.; Ros, R.; Anselmetti, D.; Reimann, P. Biophys. J. 2006, 90, 3851-3864. 
Table 2. Comparison of Kinetic Parameters for Dissociation between Linear Alkanes in Water from This Work and Results Obtained by Histogram Fitting ${ }^{a, b}$

\begin{tabular}{|c|c|c|c|c|c|c|c|}
\hline \multirow[b]{2}{*}{ model } & \multirow[b]{2}{*}{ parameters } & \multicolumn{2}{|c|}{$\mathrm{C}_{14} \mathrm{H}_{29^{-}}$} & \multicolumn{2}{|c|}{$\mathrm{C}_{16} \mathrm{H}_{33^{-}}$} & \multicolumn{2}{|c|}{$\mathrm{C}_{18} \mathrm{H}_{37^{-}}$} \\
\hline & & this work & previous work $^{c}$ & this work & previous work $^{c}$ & this work & previous work $^{c}$ \\
\hline B-E & $\begin{array}{l}x^{\star}[\mathrm{nm}] \\
k_{0}\left[\mathrm{~s}^{-1}\right] \\
\Delta G^{*}[\mathrm{~kJ} / \mathrm{mol}]\end{array}$ & $\begin{array}{l}0.30 \pm 0.06 \\
5.2 \\
36.1 \pm 0.6\end{array}$ & $\begin{array}{l}0.45 \pm 0.02 \\
0.4 \\
41.9 \pm 0.3\end{array}$ & $\begin{array}{l}1.3 \pm 0.2 \\
0.01 \\
52 \pm 3\end{array}$ & $\begin{array}{l}0.73 \pm 0.05 \\
0.02 \\
49 \pm 2\end{array}$ & $\begin{array}{l}1.00 \pm 0.13 \\
0.28 \\
43 \pm 1\end{array}$ & $\begin{array}{l}0.47 \pm 0.01 \\
0.5 \\
42.4 \pm 0.3\end{array}$ \\
\hline cubic & $\begin{array}{l}x^{*}[\mathrm{~nm}] \\
k_{0}\left[\mathrm{~s}^{-1}\right] \\
\Delta G^{*}[\mathrm{~kJ} / \mathrm{mol}]\end{array}$ & $\begin{array}{l}0.32 \pm 0.05 \\
5.0 \\
36.2 \pm 0.4\end{array}$ & & $\begin{array}{l}1.6 \pm 0.2 \\
0.004 \\
54 \pm 3\end{array}$ & & $\begin{array}{l}1.20 \pm 0.13 \\
0.21 \\
44 \pm 1\end{array}$ & \\
\hline cusp & $\begin{array}{l}x^{*}[\mathrm{~nm}] \\
k_{0}\left[\mathrm{~s}^{-1}\right] \\
\Delta G^{*}[\mathrm{~kJ} / \mathrm{mol}]\end{array}$ & $\begin{array}{l}0.33 \pm 0.05 \\
5.0 \\
36.2 \pm 0.3\end{array}$ & $\begin{array}{l}0.59 \pm 0.03 \\
0.28 \\
43.8 \pm 0.7\end{array}$ & $\begin{array}{l}1.8 \pm 0.2 \\
0.002 \\
56 \pm 3\end{array}$ & $\begin{array}{l}0.97 \pm 0.08 \\
0.016 \\
51 \pm 2\end{array}$ & $\begin{array}{l}1.30 \pm 0.14 \\
0.18 \\
44 \pm 1\end{array}$ & $\begin{array}{l}0.64 \pm 0.02 \\
0.19 \\
44.8 \pm 0.5\end{array}$ \\
\hline
\end{tabular}

${ }^{a}$ Reported errors calculated using the covariance matrix. ${ }^{b}$ The Arrhenius pre-exponential factor is assumed to be $10^{7} \mathrm{~s}^{-1} .22 c$ Results obtained from fits of rupture force histograms. ${ }^{22}$

obtained here indicate that it is likely that this collapsed state is not unique but might comprise a different geometrical arrangement of alkanes.

\section{Conclusions}

Here we have considered the detection probability of rupture events in force spectroscopy when the probability to form a molecular bond is independent of the probe velocity. The developed model indicates that for a noise-limited detection the probability as a function of probe velocity might have a maximum at a particular value of probe velocity (optimal detection velocity). Furthermore, this optimal detection velocity is independent of dissociation rate and depends on the width of the potential barrier to dissociation and on noise characteristics of the instrument. This model explains the appearance of the maximum in the detection probability reported previously in force spectroscopy measurements of hydrophobic interactions. ${ }^{22}$ Also, it is proposed that detection probability dependence on probe velocity can be used to extract kinetic parameters of dissociation. In contrast to the probability-based method proposed by Dudko et al, ${ }^{30}$ the method used here allows straightforward incorporation of simultaneous rupture of two molecular bonds. This is achieved by using the specific models of force-driven dissociation kinetics. When applied to measurements of biotin-streptavidin interactions, this method gives kinetic parameters similar to parameters obtained using other methods. In contrast, the results obtained for interactions between linear alkanes in water indicate possible structural heterogeneity of the bound state. We also note that this method can be used to estimate kinetic parameters for dissociation between molecules with relatively high dissociation rates where application of the loading rate-based data analysis approach is hindered. These might include other specific (e.g., receptorligand, antibody-antigen, enzyme-inhibitor, etc.) or nonspecific interactions.

Acknowledgment. The authors are grateful for financial support from NSF Grant CHE-0719043.

Supporting Information Available: Derivation of analytical result for the optimal detection velocity using the approximate FJC model. This material is available free of charge via the Internet at http://pubs.acs.org. 